\title{
Raconter le printemps arabe, entre fiction et urgence: \\ Une étude générique des témoignages littéraires égyptiens parus depuis 2011
}

\author{
Chaaban El Sayed Abdel Latif \\ Maître de conférences à l'Université du Fayoum
}




\section{Raconter le printemps arabe, entre fiction et urgence:}

\section{Une étude générique des témoignages littéraires égyptiens parus depuis 2011}

Chaaban El Sayed Abdel Latif

Maître de conférences à l'Université du Fayoum

\section{Résumé :}

La présente étude a pour objectif de valoriser l'apport des récits égyptiens à caractère testimonial, écrits suite à la vague de contestations qui a balayé massivement l'Egypte dès le début du 2011. On va, tout d'abord remettre en question un certain nombre de notions telles que: printemps arabe, révolte et révolution, écriture d'urgence et frontières génériques. On va, ensuite, analyser les différentes caractéristiques et distinctions génériques de ces écrits et s'ils relèvent tous de l'écriture "d'urgence". Ces derniers sont à mi-chemin entre le réel et le fictif. Ils n'appartiennent pas au même genre littéraire. Nous étudierons donc, dans un premier temps, ces récits dans leur rapport à la fiction; puis ce serait au rapport Histoire/fiction ou intertextualité (référence à la multitude d'écrits sur les dix-huit jours qui ont précédé la démission de Moubarak). Nous allons nous intéresser, par la suite, à la représentation de La Place Tahrir en tant que lieu symbolique dans les textes choisis à laquelle renvoie la dualité ou la tension : jour et nuit, intérieur et extérieur et dedans et dehors...etc

Descripteurs: Printemps arabe- Récits égyptiens- RévolteRévolution- Ecriture d'urgence- Genre littéraire- FictionTémoignages- Place Tahrir

ملخص البحث: تهدف هذه الدراسة إلي تقييم ما ساهمت به الكتابات المصرية التي تنتمي لما يسمي بأدب الثهاده والتي تمت كتابتها في مصر عقب حركات التهات الاحتجاج 


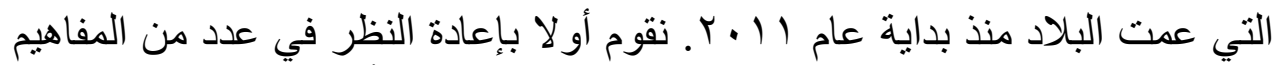

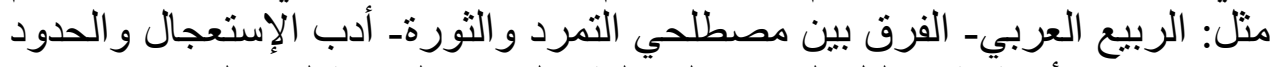

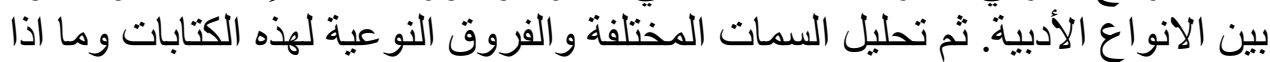

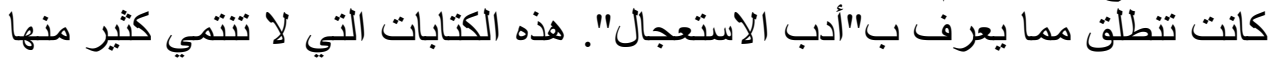

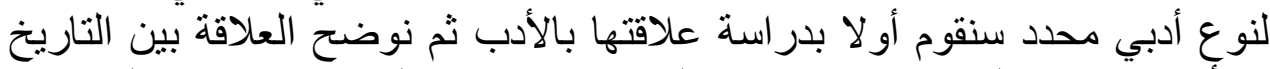

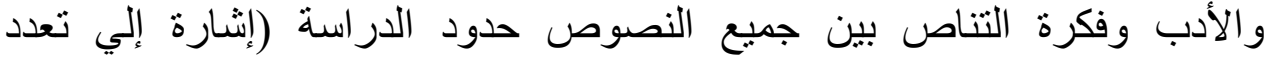

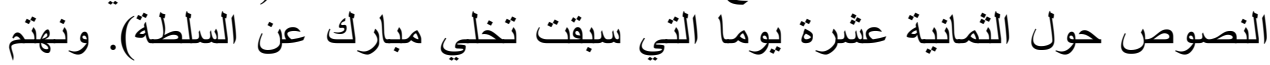

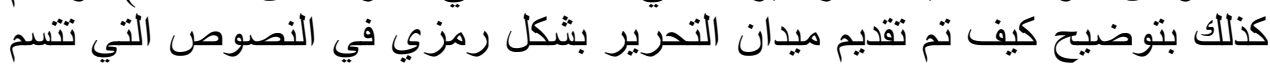

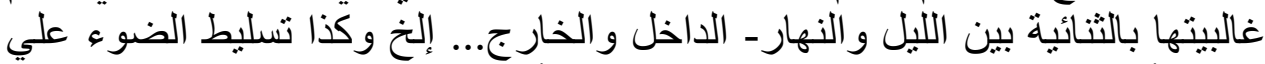

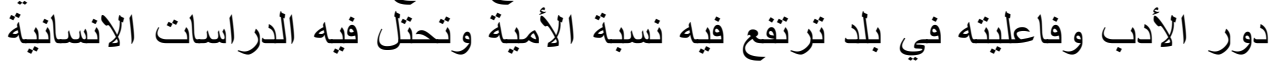
و الاجتماعية مكانا هامشيا.

الكلمات الدالة: الربيع العربي- التمردـ الثورة- الأدب المصري- أدب الاستعجالأدب الثهادة- ميدان التحرير التحري

\section{Introduction}

Le concept du «printemps arabe » né à l'hiver 2010/2011, a été curieusement inventé par certains politologues, intellectuels et journalistes occidentaux ${ }^{1}$ pour décrire un immense mouvement protestataire qui a embarrassé certains pays du monde arabe comme la Tunisie, l'Egypte, La Lybie, Le Yémen et La Syrie. En fait, il s'agissait d'un cliché qui circulait partout et qui faisait largement penser au « printemps

\footnotetext{
${ }^{1}$ L'historien américain Daniel Pipes, spécialiste du Proche- Orient a posté en mai 2011 un bref article intitulé «Arab Spring»-Misnomer, où il conteste largement ce concept, dans lequel il explique que d'abord le soulèvement n'a pas commencé au printemps, et qu'ensuite le terme est forcément optimiste, alors qu'il ne correspond pas à la réalité et l'atrocité des choses survenues pendant les dernières années.
} 
des peuples » de $1848^{2}$ en Europe et on entend même certains historiens parler d'une similitude entre les deux mouvements ${ }^{3}$.

Quant à la notion du «printemps arabe », elle se réfère notamment à un paysage nouveau et à un contexte différent. En effet, les premiers signes concrets de ce changement sont apparus dans les arts ${ }^{4}$, la langue ${ }^{5}$ et la littérature. Une vaste réflexion a eu lieu sur la façon dont le 25 janvier 2011 a été transfiguré et raconté dans les témoignages littéraires égyptiens produits après coup. Une véritable activité artistique et littéraire accompagnait les tumultes politiques et sociaux dans les places publiques urbaines et cela pourrait être partiellement interprété par la présence active de nombreux intellectuels, artistes, écrivains ainsi qu'une part significative de la jeunesse, la vraie force de cette révolte. Ces événements

\footnotetext{
${ }^{2}$ Une série de révoltes populaires ont éclaté en Europe. En février 1848, à Paris, les libéraux se sont soulevé contre le roi Louis-Philippe; après quelques semaines de révolution, il a abdiqué.

3 L'historien Benjamin Stora parle de cette similitude «une volonté identique de se réapproprier une liberté individuelle» in http://www.slate.fr/story/34563/printemps-peuples-revolutions-arabe.

${ }^{4} \mathrm{~N}$ 'oublions pas que les soulèvements populaires qui ont eu lieu en Egypte ont été marqués par une forte dimension artistique). Il y avait une omniprésence dans les espaces publics urbains (spécialement dans les rues et sur les murs) de graffiti grâce aux jeunes activistes et aux artistes. Ces graffiti étaient largement marqués par l'humour en dépit de souffrances et d'oppressions. Là, on constate une absence nette d'idéologie unique. Les murs devenaient le symbole de liberté conquise et presque absolue.

${ }^{5}$ Durant les manifestations, les grèves et les marches collectives, la langue et les mots étaient une arme qui attribuait une nouvelle dimension au phénomène traditionnel de la révolution. Elle avait un pouvoir contestataire efficace en énonçant des slogans très attirants afin d'instaurer le changement radical social et politique. Plusieurs études linguistiques ont été faites sur les slogans révolutionnaires égyptiens, on cite à titre d'exemples: Etude linguistique du slogan révolutionnaire égyptien (La révolution du 25 janvier 2011) un master en sciences du langage fait par Yasser Abdel Aziz à l'Université Mentouri de Constantine, soutenu en 2011.
} 
ont, par la suite, donné envie aux écrivains acteurs ou témoins de prendre plume depuis le déclenchement de ces bouillonnements en décembre 2010. Ils se mettaient à raconter ce qu'ils ont vu ou ce qu'ils croyaient. Ceci a donné naissance à une grande série de textes particulièrement vastes et d'une variété remarquable: romans, poèmes, essaies, autobiographies, blogs...etc. On assiste particulièrement à un retour de force des écrits à caractère à la fois testimonial et autobiographique.

Une immense difficulté est à élucider, à partir de cette étude, concernant l'utilisation des termes révolution et révolte en se référant à la situation en Egypte après le 25 janvier 2011. Il faut se méfier des interprétations trop enthousiasmées voire inexactes qui voient dans les évènements des 18 jours qui vont du 25 janvier jusqu'à la démission de Moubarak le 11 février 2011, une révolution au sens propre du terme. La question ne peut être tranchée qu'à posteriori, vu les résultats produits. Nous nous contenterons de parler de « révoltes », terminologie assez vague qui a l'avantage de ne préjuger en rien les processus ultérieurs. L'historien Jean Tulard définit, en prenant l'exemple de l'Égypte, le concept de "révolte qui est un mouvement local et spontané qui se mate par la répression ou en donnant satisfaction aux révoltés. Quant à la révolution, elle est menée par une catégorie sociale qui n'a pas sa place dans la société et s'appuie sur des mouvements populaires. Elle aboutit à la transformation des institutions et des hommes ${ }^{6} »$.

\section{La révolte égyptienne, était-elle littérairement attendue?}

\footnotetext{
${ }^{6} \mathrm{http} / / /$ education.francetv.fr/videos/jean-tulard-1-egypte-revolte-ourevolution-v109820
} 
Etudier l'efficacité de la littérature dans son rôle social est très discutable dans un pays où les études humaines en règle générale et la littérature en particulier ne sont pas prises au sérieux et occupent une place tout à fait marginale. Un pays fortement marqué par l'analphabétisme ${ }^{7}$. Ceci a à voir avec les d'habitudes de lecture et la place que la lecture et la littérature occupent dans le quotidien des Egyptiens (la lecture est essentiellement réservée aux professeurs des universités, aux écrivains, aux journalistes). Donc, on ne peut pas aller jusqu'à dire que les récits de témoignage ont directement contribué à préparer le peuple égyptien à se réveiller et à se lever contre le régime oppressant de Moubarak. C'est seulement quand ces œuvres sont transformées en films cinématographiques qu'elles deviennent massivement efficaces vu le rôle important joué par le cinéma dans la vie quotidienne des Egyptiens.

Tout ceci nous amène à dire que cette révolte était littérairement inattendue ${ }^{8}$ et insaisissable. Personne n'a vraiment prédit l'éruption de ce qu'on a appelé « le printemps arabe ». Ni politiciens ni écrivains n'ont prévu les événements qui se sont déroulés pendant l'année 2011. Les écrivains égyptiens étaient particulièrement impuissants littérairement à prophétiser ce genre d'effervescences ou de révoltes d'une manière claire ou même lui donner une nomination. De même, en nous penchant sur l'histoire et la sociologie du phénomène

\footnotetext{
${ }^{7}$ Le taux de l'analphabétisme, selon les études statistiques de recensement, atteint plus de $30 \%$.

${ }^{8}$ Là, on contredit la thèse qui dit que dans ces mouvements « rien n'était inattendu » et va même jusqu'à dire qu'il y a des textes annonciateurs ou prémonitoires qui ont prophétisé ces soulèvements massifs comme si les écrivains étaient des visionnaires. Nous constatons que certains écrivains ont même démenti le caractère prophétique ou la fonction augurale de leurs œuvres tels que Mohamed Salmawi dans Ajnihat al farasha ou Les Ailes $d u$ papillon (traduit récemment par Mona Latif Ghattas et Jaqueline Jondot, Orients Editions, 2015). En fait, on a un regard critique vis-à-vis ce genre d'affirmations.
} 
universel de révolution, nous constatons que la prévision d'une révolution ${ }^{9}$ du point de vue social, est presque impossible selon de nombreuses études publiées dans ce domaine. Pierre Favre a discuté cette question de l'imprévisibilité d'une révolution dans l'ordre social. Retenons-en, ici, une conclusion significative : « s'agissant du futur, on se trouve face à un processus non achevé, en cours d'effectuation, et dont la connaissance ne peut, par nature, qu'être inachevée. La prévision n'est donc pas possible ${ }^{10} »$.

Le chercheur n'est donc pas d'avis qu'il y avait des textes qui étaient directement annonciateurs de ces évènements étonnants de transformations qui ont bouleversé la scène socio politique, voire intellectuelle. Certains écrivains démentaient même le caractère avant coureur de leurs œuvres. On cite, à titre d'exemple, Mohamed Salmawy qui a écrit Agenehat AlFaracha ou Les ailes du papillon, œuvre connue comme ayant décrit de façon prophétique l'atmosphère explosive à la fin du règne de Moubarak. Les scènes de révolte qu'on a vu se dérouler dans les rues du Caire sont décrites avec une incroyable clairvoyance annonciatrice: manifestations houleuses, sit-in, insurrections, mais aussi solidarité de l'armée avec le peuple. Là se chevauchent le fictionnel et le factuel. L'auteur des Ailes du papillon, Mohamad Salmawy, s'étonne lui-même de ses prophéties :

\footnotetext{
${ }^{9}$ Il y a plusieurs publications sur cette question, citons à titre d'exemples Nikki Keddie, "Can Revolutions Be Predicted ? ", dans Iran and the Muslim World, New York, New York University Press, 1995, p. 13-33 ; Jeff Goodwin, No Other Way Out, Cambridge, Cambridge University Press, 2001 ; François Chazel, « La place de la mobilisation dans une révolution inattendue. L'effondrement de l'Allemagne de l'Est », dans $D u$ pouvoir à la contestation, Paris, LGDJ, 2003, p. 163-192.

${ }^{10}$ Favre, Pierre Comprendre le monde pour le changer, Paris, Presses de Sciences Po, 2005.
} 
"J'ai fini mon roman à la fin de l'été et il est paru quelques semaines avant que n'éclatent les événements en Tunisie! »Il ajoutait «La révolte m'a surpris malgré Les ailes du papillon ». Il a même avoué très clairement : "Je ne voulais pas prévoir l'éclatement d'une révolte, mais j'étais préoccupé par la situation politique qui régnait en Egypte et qu'on appelait poliment "mouvement politique». Et il a ajouté "Mon roman dont l'écriture a duré une année et dont la deuxième édition va bientôt expirer, n'a pas du tout servi la révolte, car il a été publié quelques jours avant son éclatement ${ }^{11} »$.

\section{Symptômes d'une révolte imprévue:}

Pourtant, il y avait de nombreux romans, publiés pendant la deuxième moitié du siècle passé, ayant pour thème la mobilisation populaire et la contestation contre la pauvreté et les abus du pouvoir. Des cuvres qui portaient en elles le ferment de la révolte et de la rébellion en lançant un véritable cri contre l'ordre établi, sans prétendre pour autant être prophétiques ou anticipatrices. La mobilisation y apparaitt comme un espace d'évasion ou de liberté. Chaque œuvre offre sa propre représentation. Mais, dans l'ensemble, elles se ressemblent, presque toutes, à décrire les mêmes souffrances et les mêmes oppressions. Ces textes ont décrit directement ou indirectement des sentiments d'injustice et les conditions insupportables qui régnaient dans le pays à savoir: les pratiques systématiques de tortures, les abus du pouvoir, la répression, le clientélisme, les inégalités sociales, les exactions policières, les oppressions, l'autoritarisme et la corruption qui sont devenus comme une monnaie courante qui

\footnotetext{
${ }^{11}$ Propos recueilli par Les Emirats aujourd'hui, le 15 mars 2011, en ligne sur http://www.emaratalyoum.com/life/culture/2011-03-15 .
} 
dominaient l'Egypte sous le règne de Nasser, Sadate et Moubarak. Par ailleurs, les écrivains mettent, depuis longtemps, dans leurs œuvres, les idées de mobilisation populaire, de contestation et de résistance balayant toutes les idées reçues sur la soumission aux tyrans.

Par ailleurs, si l'image du peuple égyptien, a bien vu une émancipation dans le roman égyptien; celle-ci n'était que partielle et imparfaite. Les intellectuels ont beaucoup souffert pendant la deuxième moitié du siècle écoulé, sous les régimes de Nasser et de Sadate. La situation des écrivains s'est relativement améliorée sous le régime de Moubarak. Mais toujours est-il que la littérature, en règle générale, était marginalisée et était considérablement marquée par son aspect apolitique plus ou moins pacifique. Mais cet aspect apolitique était, lui aussi, considéré comme forme de politique par les autorités et plusieurs œuvres ont fait l'objet de vandalismes et de censure.

Et parmi ces ouvrages qui illustrent la scène pleine d'injustice et de répression, on cite:

1) Chicago $^{12}$ (2007) d'Alaa El Aswany qui décrit la vie des étudiants arabes boursiers à l'Université de biologie de Chicago aux États-Unis après les événements du11 septembre 2001. El Aswany attaque très durement le gouvernement égyptien et dès la première ligne le dictateur Moubarak. Il dresse, dans son roman, un portrait accablant de la torture, de la corruption, du déficit de l'avenir, des promotions injustifiées et de la dictature. Bref, ce roman est un cri d'alarme pour dénoncer Moubarak et toute sa clique: il met en évidence tous les maux qui rongent la société égyptienne contemporaine à savoir : islamisme, misère sexuelle, violence,

${ }^{12} \mathrm{El}$ Aswany, Alaa Chicago, traduit de l'arabe par Gilles Gauthier, Actes Sud, 2007. 
intolérance, question de l'exil, de l'appartenance et des origines.

2)Le roman de Khaled El Khamissy et qui s'intitule $\operatorname{Taxi}^{13}$ (2006) où le romancier regroupe, en une quasi enquête de terrain mêlée de création littéraire. Il s'agit de cinquante-huit conversations avec des chauffeurs de taxi du Caire. Ces conversations dressent un tableau de l'Egypte à un moment décisif (avril 2005-mars 2006) du règne du président Hosni Moubarak, l'année où il a sollicité un cinquième mandat. On a deux ou trois pages chaque fois qui illustrent la colère des Egyptiens et leur ressentiment vis-à-vis du régime Moubarak, entre 2005 et 2006: un raïs au règne interminable, qui a su se perpétuer au pouvoir grâce à la brutalité de ses services de sécurité et la complicité de ses alliés internationaux. Alors, on se moque de lui dans la rue, faute de pouvoir s'en débarrasser. Tout anticipe et annonce une révolution sans le dire ou le nommer clairement. On a également l'importance donnée à la place Tahrir, comme un lieu symbolique.

3)Vient en troisième lieu le roman de Mohamed El Fakharany, Fassel lel Dahsha (un intermède ... pour l'étonnement) ${ }^{14}$ (2006). L'auteur présente l'univers des bidonvilles, des personnages marginalisés et oubliés dans les taudis pour révéler des moments humains intensifiés. Un texte révélateur à plusieurs niveaux : narratif et thématique. Il essaie de frayer un nouveau chemin singulier dans l'écriture romanesque pendant la dernière décennie et n'a de cesse qu'il n'affronte les limites du dicible sur la marginalité. Son œuvre emprunte des modes d'écriture variés pour tracer un parcours spécifique à travers les domaines du langage, de la société et de la politique.

13 Al-Khamissi, Khaled Taxi (traduit de l'arabe par Moïna Fauchier Delavigne et Hussein Emara), Arles, Actes Sud, 2009.

14Mohamed Al-Fakharani, Fassellildahcha (intermède ... pour l'étonnement), éditions Al-Dar en 2006. 
4)De plus, dans Istasia ${ }^{15}$ (2010) de Khairy Shalaby, se manifeste les maux à l'origine de la révolte égyptienne à savoir la corruption, la relation musulman/ chrétien ou l'unité nationale. Istasia, une veuve paysanne copte dans le delta égyptien, qui consacre sa vie à venger la mort de son fils unique à travers la prière. Elle sera aidée par le fils d'une famille musulmane très influente du village. Cela pose la question de l'unité nationale et la lutte contre les corrompus et les faux puissants.

De même, plusieurs autres œuvres, notamment celles qui appartiennent à ce que l'on pourrait appeler « la littérature carcérale ${ }^{16}$ ont décrit les sentiments du désespoir et de misère qui étaient à la base de ces soulèvements populaires. Ces écrivains étaient, pour la plupart de gauche, et ont été emprisonnés pour des raisons politiques et idéologiques. Cette écriture carcérale varie entre une écriture directe à caractère informatif et documentaire et une autre plus ou moins fictionnelle. Tout ceci nous amène, en fin de compte, à retenir la conclusion qui dit que rien n'était prévu au niveau fictif.

\section{Les témoignages littéraires égyptiens et l'écriture d'urgence.}

Nous interrogeons les premiers témoignages littéraires écrits sur la révolte égyptienne pour mettre en évidence que ces

15KhaïriChalabi, Istasia, éditions Dar al-Chourouq, 2010.

16 Il s'agit des témoignages sur le milieu carcéral. Des récits individuels et d'autres à caractère collectif qui décrivent le quotidien de la prison avec vérité et, parfois même, avec humour. Leur imagination les soutient, ainsi que le brûlant désir de raconter, leur seule, leur véritable arme de survie. Un voyage à travers l'expérience carcérale. Un voyage qui passe par la perte de ses droits, de sa propre humanité, un "voyage en enfer » où chacun de ces écrivains a trouvé une manière de s'échapper et de survivre. (Voir Chaaban El Sayed Abdel Latif, Esthétique du récit carcéral: une étude comparée, une thèse de doctorat non publiée, soutenue à l'Université du Caire, 2012) 
événements ont réellement existé, que des vies ont été passées au feu des événements et de l'écriture, que des façons de vivre et d'agir ont été modifiées. Une écriture est perçue comme un travail nécessaire et urgent. L'urgence peut-elle dès lors s'envisager comme une puissance configuratrice de sorte que 《le livre déferle en emportant tout sur son passage ${ }^{17}$ ou porte-t- elle toujours une connotation péjorative? Certains textes ont été écrits dans l'urgence, comme pour simplement réagir aux perturbations qui ont bouleversé le pays, avec une volonté d'être grâce à la production des écrivains acteurs ou témoins oculaires de ces mouvements. Mais avant d'aller dans des généralisations qui pourraient être abusives, nous partons d'une série de questions qui préciseraient les différents jalons de notre analyse :

- A quel point les textes écrits sur la révolte égyptienne s'inscrivent-ils dans une écriture d'urgence?

- L'urgence aboutit-elle, nécessairement et de manière réductrice, à une écriture circonstanciée de la révolte égyptienne?

- S'agit-il des textes de mérite littéraire malgré leur immédiateté apparente ou simplement d'œuvres opportunistes et prédatrices?

- Pourrait-on proposer une autre définition de l'écriture d'urgence à la lumière de l'analyse des textes écrits sur la révolte égyptienne?

Afin de répondre à toutes ces questions, on devrait bien définir ce concept clé et illustrer les caractéristiques formelles qui lui sont associées. L'urgence est

"L'urgence est un bon stimulant quand on écrit : il ne s'agit pas d'écrire vite, mais de saisir les faits au vol et de les mettre en mots. Toute écriture est une forme de réponse à une urgence; on n'écrit pas dans la lenteur de torpeur, du moins

Selon la définition que donne Jean-Philippe Toussaint de l'urgence (L'urgence et la patience, Paris, Les Éditions de Minuit, 2012, p. 45). 
ai-je toujours été emporté par mes personnages » nous dit Tahar Ben Jellon ${ }^{18}$. Nous constatons que ce mode d'écriture ne prend pas forcément en compte «la distanciation temporelle, le recul nécessaire pour transposer, romancer et interpréter un événement ou une circonstance exceptionnelle à savoir une révolte. C'est une écriture qui qualifie les textes qui répondent à une situation immédiate ${ }^{19} \gg$.

Dans ce mode d'écriture, se pose également la question du genre littéraire surtout celui de l'autobiographie : "L'écriture d'urgence aurait nécessairement des implications formelles, permettant de défier les démarcations génériques ${ }^{20} \%$. On trouve confirmation de ceci dans "L'œuvre d'urgence, paradoxalement, porte alors comme une nécessité la justification autobiographique, un positionnement de l'écrivain à l'intérieur de l'événement car, ne parlant pas en vertu de son art, l'artiste est privé de tout statut. Dans l'urgence, il est donc toujours illégitime comme "je» individuel et sa légitimité va se fonder dans la dilation du «je» en communauté. La dimension autobiographique de l'œuvre d'urgence n'a donc jamais les finalités d'une autobiographie mais sert à insérer l'artiste dans quelque chose qui le dépasse ${ }^{21} »$. Ainsi, la question du rapport de l'auteur à l'écriture autobiographique est remise en cause dans ces écritures d'urgences. L'écrivain dans cette écriture d'urgence, devient un témoin " extérieur" ou "oculaire" de son

\footnotetext{
${ }^{18}$ Propos recueilli Georgia Makhlouf dans L'Orient Littéraire, Numéro 84, 2013- 06

${ }^{19}$ Soulet, Jean François, L'Histoire immédiate, Paris, PUF, coll. «Que saisje ?», n²841, 1994.

${ }^{20}$ Fisher, Dominique, Ecrire l'Urgence, Assia Djebar et tahar Djaout, Paris, L'Harmattan, coll. »Etudes transnationales, francophones et comparées », 2008, p. 12.

${ }^{21}$ Auger, Clément, De l'urgence à la satire ancienne: autour d'une littérature en rapport avec l'événement, revue Ad hoc, $\mathrm{n}^{\circ} 2$, «l'Urgence », publié le 06/06/2013 (en ligne) URL : http://www.cellam.fr/?p=4133, p.5.
} 
temps, il partage l'espace-temps de ses contemporains dont il se fait le porte-voix. Les textes sont écrits hors de tout format fixe, aux frontières de la fiction, du récit, du récit de paroles, de l'autobiographie et de l'historiographie 22 : "La faculté de se souvenir relève de l'individuel, mais certains contenus de cette mémoire coüncident avec une mémoire plus vaste; collective et marquée par l'Histoire, la culture et les pratiques sociales, et composée de relations mutuelles et complémentaires entre mémoires individuelles ${ }^{23} »$.

Dans l'écriture de l'urgence, la mémoire peut revêtir une autre forme, celle du "devoir de mémoire". L'écrivain se détache d'une assignation à l'instant en tentant de recouvrer les mémoires forcloses dont procèdent les situations extrêmes ${ }^{24}$. Ainsi, cela se présente sous forme d'un cri pour tenter de constituer urgemment une mémoire collective.

L'urgence est également en corrélation étroite avec l'écriture testimoniale. L'écriture de témoignage et sa définition comme écriture d'urgence. Dans l'écriture testimoniale, la réaction que constitue l'écrit "possède une dimension fondamentalement éthique, c'est une façon de récupérer sa dignité d'être humain ${ }^{25} »$. De même, l'accent est mis sur le caractère collectif d'une écriture d'urgence : "Une urgence se vit collectivement. Dans le cas contraire, l'auteur cède à une pression artificielle, la fausse urgence, l'empressement ${ }^{26}{ }^{2}$.

Donc et suivant une réflexion hypothétique, nous admettons d'abord que l'écriture sur ces effervescences s'inscrit, en principe, dans le cadre d'une écriture d'urgence qui intervient

${ }^{22}$ Dominique Fisher, Écrire l'urgence, Assia Djebar et Tahar Djaout,., Paris, L'Harmattan, 2008 p. 18.

${ }^{23}$ Ibid.,

${ }^{24}$ Ibid., p. 84.

${ }^{25}$ Estripeaut- Bourjac, Marie, L'écriture de l'urgence en Amérique Latine, Pressac, Presses Universitaires de Bordeaux, 2012, p. 60.

${ }^{26}$ Auger, Clément, op. cit., pp. 10-11. 
même dans une situation de révolte. Des écrits parus après coup, quasiment dans la hâte ont une valeur documentaire indéniable. Une «écriture de circonstance » est loin de faire l'unanimité du point de vue qualité esthétique ou formelle en raison du manque de recul nécessaire à la maturation d'une œuvre.

D'ailleurs, pour affiner notre analyse, il nous convient de percevoir les particularités génériques des textes choisis, avec notamment le retour de force des écrits à caractère autobiographique avec leur variété et leur richesse. Donc, pour que cette analyse soit méthodologiquement bien fondée et profondément construite, on va essayer de répondre à ces deux questions fondamentales :

- Quelles sont les différentes manifestations de la révolution dans ces œuvres dites d'urgence?

- Quelles sont leurs différences majeures du point de vue générique?

\section{Ecriture d'urgence et barrières génériques: analyse et perspectives}

Il est remarquable que depuis cinq ans fleurissent des écrits, d'une immense diversité, tentant d'appréhender une réalité égyptienne mouvante, controversée, explosive et ambigüe. Cette révolte, on la voit dans tous nos textes. Pour les uns, Il s'agit d'une certaine insistance et d'une nécessité de fixer, d'engranger ce qui va devenir du passé. Ces écrits relèvent de genres différents (Chroniques, témoignages, récits de vie, entretiens, essais, études, romans, poèmes...etc) et sont de qualité inégale. Se différenciant par le genre et la qualité, ils ont un dénominateur commun : L'Egypte en ébullition. Des écrits qui révèlent l'ampleur d'une révolte dans le but d'immortaliser cet instant rare et important où un peuple affirmait sa souveraineté. De manière générale, cette période se caractérise par une floraison d'écritures. Des écrivains «encouragés» par la 
situation de leur pays, ont recours à l'écriture de témoignages ou de textes fictionnels. L'expression du témoignage, plus directe, plus spontanée que celle du texte purement fictionnel, est propre à des auteurs submergés par la douleur et la révolte et ne se préoccupant pas de les filtrer à travers une forme "élaborée ». En fait, cette constatation n'est pas générale et définitive. Elle est ponctuelle, liée aux premiers textes qui sont seulement écrits à chaud, d'où se posait la question d'écriture d'urgence ou d'écriture événementielle immédiate. Ces textes appartiennent, diversement, à ce que l'on pourrait appeler" l'écriture immédiate de l'évènement "27 ou " l'écriture de circonstance ou de crise".

On envisagera, essentiellement, les textes qui nous paraissent fondateurs notamment du point de vue catégorisation générique. Des textes écrits, pour la plupart d'entre eux, dans la chaleur de l'Egypte de 2011, à la surprise de tout le monde, un jour du janvier a complètement bouleversé l'Egypte, tombée dans le désespoir et les oppressions par plus d'un demi-siècle de dictatures. Entre l'historique et l'autobiographique, le fictionnel et le factuel, les écrivains parlent, dans leurs œuvres, d'une révolte au quotidien et l'observent avec un regard ému, engagé, ironique ou auto ironique même. Ils y réévaluent l'histoire égyptienne moderne et l'auto image nationale, sans les euphémismes de la censure ni l'irréalité imaginaire d'une Egypte sous les dictatures qui vont de Nasser à Moubarak et qui se désintègre définitivement. De même, ils y évoquent la vie collective des auteurs qui jouent le rôle des historiens du quotidien en quelque sorte ; ceux qui font "la petite Histoire" au jour le jour. En bref, ceux qui ont une vraie fonction sociale.

27 On fait allusion, essentiellement ici, à Roland Barthes, dans son article important intitulé «L'écriture de l'événement » où il se pose les questions : comment un événement peut-il être écrit? Et qu'est-ce que cela peut vouloir dire «une écriture de l'événement » ?- Communications, 1968, Volume 12, Numéro 1, pp. 108- 112. 
Ainsi, au lendemain des mobilisations, paraissent plusieurs œuvres, de qualité littéraire variée, qui reflètent largement le retour en force de plusieurs sous genres: autobiographique, semi autobiographique ou semi historiographique de prétention littéraire (on pourrait même le constater à travers les titres : Yawmiyyat... Awraq... Kurrasa ou même Ayyam). Nous proposons ici une analyse générique de quelques-uns de ces textes. Si le point du départ, dans tous ces écrits, est un ensemble d'impressions sur le quotidien de la révolte égyptienne. Le travail d'écoute des voix, en ce moment d'euphorie est sûrement l'un des points les plus séduisants dans plusieurs textes (surtout quand il s'agit des auteurs témoins oculaires comme Hicham el Khishin dans Sept jours à Tahrir ${ }^{28}$, Ibrahim Abdel Meguid dans Les jours de Tahrir ${ }^{29}$, Mona Prince dans Je m'appelle Révolution ${ }^{30}$ et Mekawi Said dans Cahier de Tahrir, des histoires et des lieux $\left.{ }^{31}\right)$, constituant d'ailleurs un choix permanent d'écriture littéraire, suivie par divers auteurs de la même période.

Ainsi, les notes ${ }^{32}$ sur les dialogues entendus dans la Place Tahrir signalent le vocabulaire d'une nouvelle Egypte où les habitudes et le langage s'affranchissent et s'agitent partout dans les médias et les réseaux sociaux. L'écriture des évènements est, sans doute, déterminé par un point d'observation, explicitement subjectif, et par les déterminations du genre littéraire explicitement discernées depuis même le titre des œuvres. Ceci

${ }^{28}$ El Khishin, Hisham, Sept jours sur la placeTahrir, Al Dar Al Masria Al Lobnania, 2011.

29 Abdel Meguid, Ibrahim Les jours de Tahrir, pour chaque terre une naissance, Dar Ahbar El Youm, 2011.

${ }^{30}$ Prince, Mona, Je m'appelle Révolution, Dar Merit, 2012.

${ }^{31}$ Said, Mekawi, Cahier de Tahrir, des histoires et des lieux, Al Dar Al Masria Al Lobnania, 2014.

${ }^{32}$ N'oublions pas que certains écrivains comme MekawiSaid gardaient toujours un block notes dans sa poche où il enregistre toutes ses remarques quotidiennes qu'il a ultérieurement publiées sous forme de kurassa. 
étant dit, il nous reste donc de nous pencher sur quelques ouvrages qui nous paraissent manifester cette variété générique.

Nous commençons par Sab'atayyam fi al- Tahrir ${ }^{33}$ (Sept jours sur la place Tahrir), connu comme premier roman qui raconte la révolte égyptienne et qui n'a pas été traduit en français, de Hisham al- Khishin où l'auteur y livre les sept clés de la révolution entre lundi 24 , la veille du « jour de la colère $»-$ le début du sit-in sur la place Tahrir et la démission de Moubarak. Le livre a été écrit rapidement et cela se manifeste dans la langue, la construction même du roman, qui relate les événements de manière rigoureusement chronologique et linéaire. Nous trouvons le mot «roman» sur la couverture du livre mais il s'agit plutôt d'un journal qui enregistre au jour le jour les événements de la révolte à travers la vie de différents personnages du 24 janvier au 11 février 2011, date du renoncement de Moubarak au pouvoir. L'auteur s'intéresse à tout dire. Le romancier, en témoin oculaire, voulait jouer le rôle de l'historien qui raconte la petite histoire du quotidien de la Place Tahrir au jour le jour. Après avoir dédié son roman (à ceux dont les cœurs jeunes ont refusé de battre que librement, aux révolutionnaires martyrs et vivants), il souligne, dans sa préface que la révolte et le romantisme entretiennent une relation d'équivalence. Il dit :

Les $\quad$ lévolutions sont

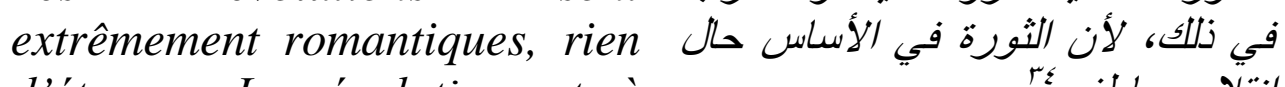
d'étrange. La révolution est, à l'origine, un état de bouleversement émotionnel

Il met, en évidence, dès le début, un élément séduisant qui, se trouve d'ailleurs dans d'autres ouvrages qui seront analysés, qui

${ }^{33}$ Sur la quatrième de couverture, outre une photo de la place Tahrir ,on retrouve le mot «roman » pour déterminer le genre d'ouvrage auquel on a à faire. On sait à l'avance, qu'il s'agit d'une œuvre fictionnelle.

${ }^{34}$ El Khishin, Hisham, Sept jours sur la placeTahrir, op. cit., p. 7. 
est l'écoute de voix, après tant d'années où tout le monde a gardé le silence :

J'ai rencontré des gens à في التحرير قابلت وتناقشت مع أناس، وبري

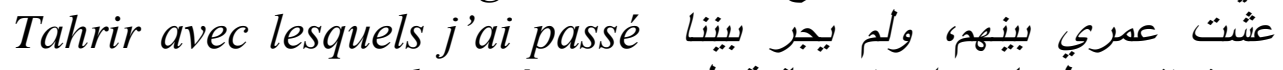

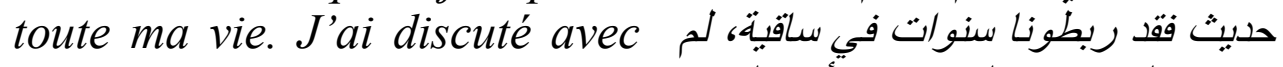

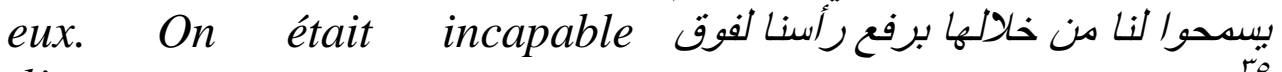
d'entretenir une conversation car on était condamné à être attaché à une roue qui tourne sans cesse. On ne nous a pas permis de lever haut la tête.

Les chapitres, (l'auteur a choisi les sept jours les plus tendus dans cette révolte qui vont du premier jour le 24 janvier au septième jour le 11 février 2011) où Il raconte comment les personnages ont vécu ces sept jours, leurs espoirs et désillusions face à la révolte, sont rythmés par les slogans cadencés lors des manifestations tantôt en arabe littéraire, tantôt en égyptien ${ }^{36}$. Dans ce roman, on constate la réunion de plusieurs personnages, habitants de la rue Hussein Hégazi à Kasr Al Aini proche de la Place Tahrir, formant une unité. Des personnages de générations, de confessions et de statuts sociaux différents et qui ont pour point commun de cohabiter dans le même quartier: «musulmans et coptes, femmes et hommes, riches et pauvres, civils ou militaires, jeunes et vieux. Comme si la Place Tahrir était libération d'un pouvoir pourri mais de tous

\footnotetext{
35 Ibid.

${ }^{36}$ Certains slogans sont dits en arabe classique comme « le peuple veut un changement de régime » الشعب يريد تغيير النظام le slogan clé de toutes les révoltes arabes, qui s'est rapidement transformé en «le peuple veut la chute du régime », الثعب يريد سقوط النظام le slogan de «dégage » إرحلest également un slogan clé dans presque tous les pays secoués des soulèvements- tantôt en arabe dialectal égyptien 《ش حنشي هو يمشي «nous ne partirons pas, c'est à lui de partir» ou encore « «Militaires, Militaires, Militaires, pourquoi ? On est en prison ou quoi ? عسكر عسكر عسكر ليه إحنا في سجن و لا ايه
} 
les complexes sociaux et psychologiques » disait Jalal $\mathrm{Amin}^{37}$, un intellectuel de gauche égyptien, en donnant son jugement sur le livre.

Par ailleurs, à chaque nouvelle apparition des personnages, le romancier les présente en fonction de leur description physique et morale, leurs motivations, leur conviction religieuse et idélogique pour participer à cet événement crucial. Ces jeunes représentent un microcosme de toute la société égyptienne. Et ce procédé couramment utilisé dans le roman égyptien moderne de présenter la société à travers les personnages d'un quartier, d'un café $^{38}$ ou d'un immeuble ${ }^{39}$. Claude Duchet explique clairement que le rôle social que le personnage romanesque pourrait revêtir est indispensable pour atteindre la cohérence et la littérarité : "Le roman est ainsi un espace imaginaire dont l'organisation relève de techniques narratives spécifiques, mais aussi (et en même temps) un microcosme social dont tous les éléments réfractent la totalité d'une unité culturelle, elle-même insérée dans le monde du réel. C'est même dans la mesure où le roman fonctionne comme une société, où il fait appel à une expérience de la socialité, qu'il atteint à la cohérence d'une pratique, et par là sans doute accède aussi à la littéraritéte ${ }^{\text {}}$.

Sept jours sur la place Tahrir témoigne ainsi d'observation de situations quotidiennes tout au long de ces jours clés qui sont plutôt transmises par un narrateur impersonnel. Le narrateur espionne son environnement. Il rapporte ainsi un ensemble de

${ }^{37}$ Il écrivait un ouvrage intitulé "Qu'est-ce qui s'est passé à la révolte

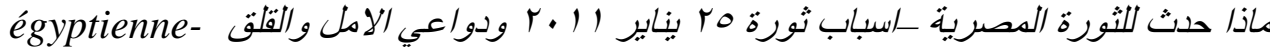
وآفاق المستقبل - عن دار الثروق

${ }^{38}$ Naguib Mahfouz l'a déjà fait dans Karnak où trois étudiants habitués du Karnak Café - qui donne son titre au livre - sont victimes des dérives dictatoriales de la révolution socialiste nassérienne.

${ }^{39} \mathrm{Alaa} \mathrm{Al}$ Aswany a déjà présenté ce microcosme dans l'Immeuble Yacoubianoù cohabitent plusieurs générations et presque toutes les classes sociales

${ }^{40}$ Duchet, Claude, Sociocritique.- Paris : Nathan, 1979, p.217. 
faits et de gestes s'inscrivant dans un temps et un univers spécifiques, Nous constatons que le narrateur occupe la place de l'observateur ou du témoin oculaire qui nous présente les représentations des vies ordinaires des personnages avec beaucoup de digressions. Les modalités narratives qui élaborent autant qu'elles bousculent les personnages sont plus ou moins mis de côté. La conjugaison entre le quotidien de ces personnages et la dynamique narrative du roman est ambiguë et imparfaite. Le romancier s'est intéressé plutôt à la perspective digressive, sociologique, ethnographique et a délaissé complètement les enjeux de la représentation d'une quotidienneté. Des scènes qui ressemblent aux descriptions journalistiques ou aux reportages d'une situation sociale et politique de certaines personnes. Le texte manque d'une certaine dynamique narrative de ce quotidien en portant toute l'attention à entasser plusieurs figures humaines de différentes convictions et de mentalités différentes dans un même espace textuel. Une volonté de tout et rien dire. Ceci a donné à son œuvre un caractère plutôt statique et immobile du point de vue formel et une appartenance générique incertaine (un récit, une nouvelle, un essai, un journal, un reportage ...?) C'est l'interférence qu'il produit avec le discours narratif.

En effet, l'écriture immédiate de la révolte dans ce texte, qui se veut fictionnel avec plusieurs dimensions testimoniales, conduit à considérer cet ouvrage génériquement comme déficient. Car, une révolte, comme elle s'inscrit dans les événements, elle devrait également s'inscrire dans l'écriture, dans le refus d'un langage traditionnel, pour échapper aux clichés, au conformisme et à l'emprise du pouvoir dont le caractère diffus en Egypte peut être insidieux. Ce qui n'est pas le cas ici. Khaled El Khishin, qui narre le plus sobrement possible le quotidien de la révolte, et qui y a trouvé un nouveau champ d'exploration thématique ; il ne l'a pas trouvé au niveau formel. Son traitement particulier du quotidien ne s'adjoint donc pas à une réflexion sur la forme de son écriture dont le caractère aplani donne à brouiller les 
frontières habituellement admises entre ce qui rompt la trame de la vie quotidienne et ce qui s'y insère avec fluidité.

En fait, les modalités de la mise en récit d'un quotidien qui précède un évènement inattendu exige le rapprochement d'un certain nombre de variables narratifs qui s'entrechoquent. Le geste de raconter appelle en effet une idée conventionnelle ${ }^{41}$. Cette idée semble peu compatible avec notre ouvrage pour plusieurs raisons tant poétiques que génériques. Car si l'on considère d'abord qu'un récit fictionnel au sens canonique du terme suppose le surgissement d'un élément ou d'un fait inattendu dans une situation mouvementée d'agitation. On doit donc remettre en doute le transgressif de l'ordinaire, dont la continuité d'un tranquille (l'horizon d'attente) écoulement quotidien manque de moment saillants surtout quand il s'agit d'une révolte qui va éclater. Le texte de Khaled el Khishin illustre cet aplanissement et ce manque de singularités ou des faits marquants. Les dialogues, dans ce texte, tiennent également au théâtre, simplement précédés par le nom du locuteur. Tout ceci accentue le caractère incertain du genre littéraire auquel appartient ce texte.

On trouve également beaucoup d'autres textes qui appartiennent aux Yawmiyyat ou Ayam et qui sont également écrits sitôt l'événement achevé, d'une littérature abondamment produite par les acteurs de la révolte. Un temps, où la littérature se propose comme moyen d'appréhension alternatif aux procédures historiques, du quotidien des révoltés sur la Place Tahrir. Et là se pose fortement la question de l'ambigüité générique.

Parmi ces nombreux écrits produits sur la révolte, viennent le texte de Mekawi Said Le Cahier de Tahrir, des histoires et des

${ }^{41}$ Cette convention renvoie à la fois à la conception structuraliste des formalistes et aux principes énoncés par Aristote dans sa Poétique (pensons au principe de l'unité et aux notions de début, de milieu et de fin) qui semblent fortement floues dans ce texte. 
lieux, qui se présente sous la forme d'un petit carnet des observations journalières de l'auteur, pour ne pas oublier les nuances les plus fines. Il a pris notes pendant les différents jours de la révolte. Ces petits détails sont ignorés de la plupart du peuple qui suivait les événements à travers les écrans de la télévision.

Mékawi Said a donc décidé de les publier enveloppés d'une écriture littéraire attirante. Il considère Le Cahier de Tahrir comme "une accumulation de petits romans, puisque chaque chapitre renvoie à un monde à la frontière du fantastique et du rée $^{42}$ ». Cette écriture est bien constituée en fragments. Ces fragments se lient harmonieusement sans problème de discontinuité narrative, spatiale ou temporelle. Et pour tenir tous les fils, le narrateur possède à la fois un regard d'ensemble et une perception de détails sur La Place Tahrir. Cette double vision singulière assure que les fragments à caractère plutôt documentaire ne tombent pas dans le désordre. Une écriture très loin d'être linéaire ou chronologique. Il s'agit plutôt d'un récit de témoignage sur des personnages marginaux, qui ont joué un rôle important mais méconnu de ceux qui ont écrit sur la révolte. L'auteur est le témoin oculaire et le garant de l'authenticité des faits:

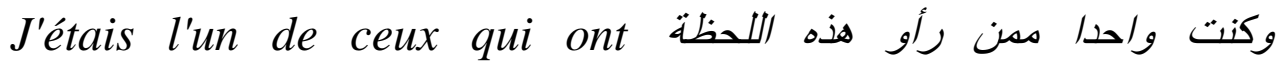
vécu ce moment et qui ont vu وعاينوا /حد/ثها ودونوا وقائعها ses actions ou ont décrit ses faits

L'évolution des personnages est vue à travers leur interaction avec un espace symbolique, un espace mythique, la Place Tahrir: circulation de ces personnages dans un espace unique. Si le récit descend souvent à des détails minutieux, mais ces circonstances, plus importantes en elles mêmes, n'attirent que partiellement son attention. En d'autres termes, les actions et les

${ }^{42}$ Entretien avec Mékawy Said publié à Al Ahram hebdo, n 1010, 2014. 
personnages sont vus par Sabrine, une adolescente de 14 ans et qui est entré La Place Tahrir tout à fait par hasard au début. Elle s'est intégrée, par la suite, avec les autres jeunes révolutionnaires et elle leur rendait toujours service. Elle était éblouie par leurs slogans. Elles se sont amassées et ont hurlé ensemble suite à l'éviction de Moubarak. Sabrine disparaît de nouveau faisant allusion au retour de la misère toujours incurable.

Dans le récit, il y a des personnages connus comme Sioufi (le tigre de la révolte) et Kamal Khalil (une figure emblématique de la gauche égyptienne) ou d'autres inconnus tels que Pierre El Siwfi, le révolutionnaire rêveur, propriétaire d'un immeuble qui donne sur le Place Tahrir et Ahmed Lotfi qui a prêté son appartement aux révolutionnaires. On peut remarquer d'autres motifs ou encore d'autres manières d'envisager un aspect du quotidien où tout se joue autour des faits et gestes sans grande importance. Le narrateur s'intéresse prioritairement à révéler le caché, le non dit des vies soustraites à l'examen avec un ton autofictif et testimonial. Le récit a pour sous titre "des histoires et des lieux" pour mettre en relief l'importance donnée à l'espace en tant que lieux symboliques avec une longue histoire.

Ibrahim Abdel Meguid, lui, dans son livre Ayyam Al Tahrir (les jours de Tahrir) (2011), pour chaque terre une naissance, publié à la hâte, ne se contente pas de nous livrer le déroulement des événements au jour le jour, mais il se raconte sous forme d'un journal intime, sans pour autant, attribuer le premier rôle aux manifestants (dont son fils fait partie) et à la Place Tahrir qui occupe le devant de la scène. Il apporte également des impressions et ses observations tout en se tenant sur le terrain. En effet, chaque chapitre met le point d'orgue sur une scène entamée dans la révolte. Mekawi Said, lui, nous l'explique; il a sous-titré son ouvrage des histoires et des lieux: "La place Tahrir et son entourage composent le centre-ville, elle est la vraie capitale de l'Egypte, puisqu'elle se situe au cour du 
Caire. A son tour, l'Egypte est la capitale du Proche-Orient. La place Tahrir est alors sensible aux événements qui s'y déroulent. Le déclenchement de la révolution a eu lieu dans cette zone, en l'exportant ensuite aux différentes places du pays. D'ailleurs, la géographie de la place, en forme de cercle avec toutes les rues et les ruelles adjacentes, a permis aux révolutionnaires d'échapper aux attaques de la police. Ajoutons aussi les appartements dont les propriétaires avaient ouvert les portes pour abriter les révolutionnaires, notamment les filles, contre les attaques,. En outre, la révolution a rendu la valeur au centre-ville, tombé dans l'oubli pour plusieurs décennies. Je parle des nouvelles générations qui avaient déserté cette zone pour le profit des nouveaux quartiers comme Al-Tagammoe AlKhamès ou les villes nouvelles à l'instar d'Al-Réhab et Madinati. ${ }^{43}$

Dans ces deux ouvrages, bien qu'ils manifestent deux plans d'énonciation différente, l'écoute et l'attente occupent une importance particulière. Il y avait une certaine hésitation voire une peur de témoigner. Cela montre qu'il a une différence énorme entre être témoin et témoigner. Témoigner veut dire passer à l'acte, faire part de qu'on a vu, entendu, et ressenti soit en position d'acteur (en prenant la parole) ou de témoin.

J'ai écrit, bien que je ne l'aie pas dans l'idée les jours de la révolte. Je me suis laissé voir, entendre et ouvrir les portes de

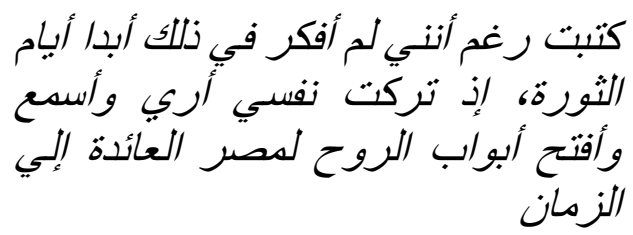
l'âme d'une Egypte qui revient dans le temps.

Par contre, Dans Plus lourd que Radwa, (avec un sous-titre :une autobiographie en fragments), on est face à texte complètement différent, au niveau poétique et stylistique, où se mêlent plusieurs genres littéraires, hésitant encore sur le genre littéraire,

${ }^{43} \mathrm{Ibid}$. 
entre chronique, mémoire et essais. Il appartient à ce que l'on pourrait appeler généralement "les écrits de for privé » ${ }^{44}$ ou bien «la littérature de l'expérience ${ }^{45} »$. Ces deux appellations désignent diversement des récits écrits par un auteur à propos de son existence et de sa perception des événements vécus, adoptant une grande variété de formes. Les différentes facettes de la romancière apparaissent de texte en texte, différentes de l'un à l'autre ou communes à certains types de texte. Elle s'y dépeinte tour à tour en professeur militante et en femme attentionnée, en témoin oculaire, et en magistrat dans l'exercice

${ }^{44}$ Depuis 2003, le Groupe de Recherches «Les écrits du for privé en France de la fin du Moyen Age à 1914 » développe une activité multiforme centrée sur ces textes si particuliers que sont les «écrits du for privé ». De même, ce champ de recherche est un lieu privilégié pour la collaboration entre historiens et littéraires, représentés ici par deux laboratoires ITEM et CRPHL, qui organisent la manifestation et coopèrent au sein du « Groupe de recherche des écrits du for privé dans les Pays de l'Adour. » $\mathrm{Ce}$ dernier, créé en 2008, a déjà à son actif une longue suite de publications et de manifestations scientifiques. En fait, ces textes sont d'une diversité assez remarquable: "Ils regroupent les livres de raison, les livres de famille, les diaires, les mémoires, les autobiographies, les journaux de toute nature (personnel ou «intime», de voyage, de campagne, de prison...) et, d'une manière générale, tous les textes produits hors institution et témoignant d'une prise de parole personnelle d'un individu, sur lui-même, les siens, sa communauté. les écrits du for privé connaissent un regain d'intérêt depuis plus d'une vingtaine d'années de la part des historiens mais aussi des littéraires, des linguistes, des sociologues, ou encore des ethnologues qui les classent dans la grande catégorie des « écrits ordinaires ».

${ }^{45} \mathrm{Il}$ s'agit, dans notre sens, des écrivains qui ont l'expérience de la maladie ou de militance, par exemple, et participent à la légitimation de cette littérature d'un genre nouveau. Une écriture qui mélanger les genres ; elle pourrait procéder de trois genres : du roman, du journal et du récit. Cette écriture a le plus souvent une narration linéaire et chronologique. Les œuvres portent parfois des sous titres qui attestent ce mélange des genres comme c'est le cas dans notre texte qui est sous-titré une autobiographie en fragments alors qu'il ne l'est que partiellement, dans le sens de celle d'une professeur militante. 
de ses fonctions. La romancière et professeur militante Radwa Achour, évoque son quotidien de la révolte où l'expérience de la maladie se chevauche avec la trame de sa vie. Cette œuvre vise essentiellement à dresser une sorte d'état présent des relations entre vie et littérature. Témoigner ${ }^{46}$ et rendre ce témoignage vivant des grands épisodes que la romancière a vécus et qui se présentent d'une manière strictement rigoureuse. Elle nous présente, dès le début, une explication du titre choisi de son autobiographie fragmentaire en disant que ce titre est inspiré d'un proverbe utilisé couramment par les arabes pour désigner une tâche lourde, douloureuse et pénible. Celui ou celle qui accomplit cette tâche doit avoir un esprit militant, une solidité, une persévérance et une opiniâtreté :

Plus lourd que Radwa est un proverbe que les arabes disent pour signifier la stabilité, la ténacité, et l'enracinement. Ils font allusion à une chaîne de montagnes proches de Médine أثقل من رضوي مثل ضربه العرب في الثيات الثبات والرسوخ، إثارة إلبي سلسلة

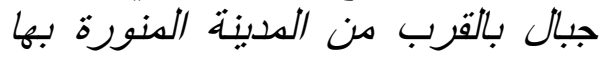

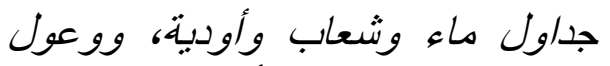
وغزلان، تحلق في أرجائها وائها النسور qui comprennent des sources d'eau, des sentiers, des vallées, des mouflons et des gazelles. Les aigles, les pigeons et les faucons y volent partout.

Le livre se présente sous forme d'un journal interrompu et incomplet (en fragments) relatant des faits qui commencent en août 2010 et se terminent le 9 mai 2013. Dans ce texte, se mêlent l'essai, le récit, l'Histoire, la géographie et les arts. Une fusion bien faite pour pouvoir témoigner, partager son

${ }^{46}$ Cet acte, outre sa valeur esthétique, pourrait avoir également une signification éthique. L'écrivain témoin, pense souvent accomplir une mission en narrant son expérience et se sent responsable de la nécessité de cette écriture.

${ }^{47}$ Achour, Radwa, Plus lourd que Radwa, (Fragments d'une autobiographie), Al-Shorouk, 2013, p. 6. 
expérience sous forme d'une autobiographie fictionnelle, de réflexions poétiques et intimes. Une œuvre de méditations personnelles, philosophiques et poétiques, met explicitement en avant sa volonté de lier son expérience intime de maladie à une expérience collective. Cette volonté est à la fois étroitement liée à la mort de son frère et sa mère. :

Il me semble évident maintenant que commencer à écrire une autobiographie, en ce temps-là, était liée d'une manière ou d'une autre à la maladie de mon frère et la détérioration de son

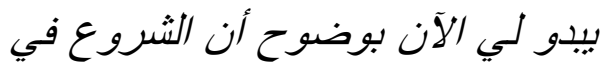

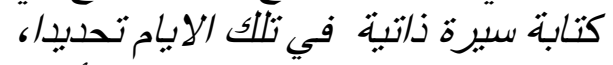

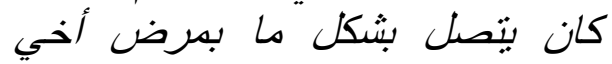

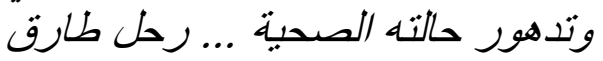

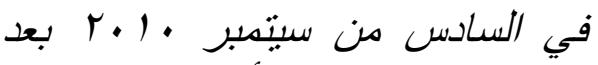
état de santé ... Tariq est mort le 6 septembre 2010 et cinq semaines plus tard ma mère l'a joint.

Elle construit, sous la forme d'un récit autobiographique fragmentaire composé de trente-trois chapitres. La romancière y raconte son expérience avec la maladie du cancer qui ne l'a pas empêchée de poursuivre, avec beaucoup de zèle, la trame de sa vie professionnelle et scientifique e tant que professeur et militante du groupe du 9 mars qui avait pour objectif l'indépendance de l'université égyptienne. On passe dans cette autobiographie de la sphère de l'intime (lutte psychologique, méditations personnelles) à une dimension plus collective à visée cognitive : témoigner, écrire pour soi mais aussi pour le monde :

Un projet d'écriture d'une autobiographie a commencé par parler de ma mère, mon père et mes frères et sours et passe par la suite à raconter l'autre part de mon récit... Il

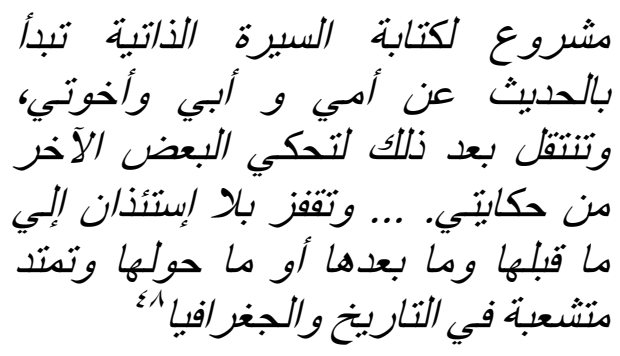
saute sans permission à l'avant

${ }^{48}$ Ibid. 
et l'entourage et va s'élargir à

l'histoire et à la géographie.

La structure de l'ouvrage illustre cette perspective. Il se découpe en trente-trois chapitres qui varient entre : la découverte du moi et qui se focalise sur l'expérience personnelle avec la maladie ou son travail à l'université d'Ain Chams et son engagement pour des causes nationales et arabes. Au-delà du drame personnel qui se déroule, c'est un témoignage qui se veut pour tout un peuple, et plus particulièrement ceux qui se sont sacrifiés pour la liberté. Cet ouvrage rend hommage à tous ceux qui ont combattu jusqu'à la mort.

De toute façon, il est important de dire que nous ne perdons pas complètement de vue la chronologie, et que nous identifions, dans l'organisation en chapitres, un travail de sélection thématique autour d'un sujet central. L'annotation et la chronique assurent la lecture des signes immédiats du présent, dans ses détails les plus variés, curieux ou insolites: L'incident du 4 novembre 2010- Un drame composé- Le septième étage d'abord- Tunis, si le peuple veut un jour- L'Egypte qui...- Je me sens quelquefois être un enfant, un repos- Le miracle de De Vinci, un retour, les morts- $\mathrm{Du}$ quatrième étage au palais Zaafran, Al Zaafran- Qui écrit cette scène ?- Sur Ahmed Al Chahat et Chaabane Mekawy- Au temps de révolution- Un petit article sur l'Histoire- La géographie et les méandres de la politique - Des embouteillages- Un petit article sur l'écritureRue de l'école Horeya- Entre autobiographie et mémoires- Sur Rhinocéros le noble- Le communiqué du carnage- Graffiti- Sur la poésie et les poètes : un chapitre qui parait hors sujet bien qu'il soir en plein sujet- L'hôpital encore une fois- L'avantpropos Claquette la cinquième fois- Une grosse souris, « $\mathrm{A}$ Master of disguise» dans le chapitre conclusif. Une construction polyphonique, une multiplicité des voix qui partagent une même expérience, tend à faire de cette expérience à savoir sa militance pour l'indépendance de l'université, une 
expérience collective que chacun peut appréhender en particulier.

\section{Conclusion}

En conclusion, les textes réunis et analysés précédemment, surmontent les enjeux génériques pour s'approcher d'une écriture qui reste à mi-chemin entre le testimonial et le fictionnel. On a vu des textes à moitié autobiographiques, à moitié fictionnels. Le témoignage dans son expression factuelle ou fictionnelle. Ces écrits revêtent un statut particulier, au croisement du réel et du fictif. Des autobiographies, des mémoires, des journaux intimes se voient néanmoins contaminées par la dimension fictionnelle de la construction narrative. Entre l'historique et l'autobiographique, les auteurs y parlent d'une révolte au quotidien et l'on a observé un regard ému, engagé et ironique. Une nouvelle écriture est donc élaborée grâce au mélange des genres. L'étude d'un corpus composé d'autobiographies, de récits fictifs, de journaux intimes, de chroniques témoigne de ces nouveaux rapports qu'entretiennent les auteurs avec leur Histoire et avec leur langue et révèle qu'une nouvelle écriture, expression d'une individualité émergente, se développe en Egypte. Libre de toute idéologie et du poids d'une histoire mal assumée, cette production littéraire est bien le signe qu'une révolte s'est produite. Comment raconter la révolte égyptienne sans tomber dans le témoignage réducteur ? C'est à cette question que la plupart de nos témoignages ont essayé de répondre. Tous ces écrits ont l'avantage d'ouvrir l'horizon à plusieurs possibles narratifs. Ils ont relativement pris l'événement révolutionnaire au chaud tout en tenant compte de ses contours qui leur étaient mal définis. Ils reflètent également le retour en force des écrits à caractère autobiographique, semi-autobiographique ou semihistoriographique sur la scène littéraire égyptienne. Les idées de révolution populaire, de mobilisation et de résistance collective, qui apparaissaient dans la production romanesque égyptienne de la deuxième moitié du vingtième siècle, préparait le terrain à 
une Egypte en ébullition et en mouvement. Cette production qui contribuait à l'émergence d'un discours révolutionnaire et subversif qui se voit clairement accentué dans les ouvrages écrits suite aux soulèvements de 2011. La question qui reste encore posée: est-ce que ces écrits vont être encore lus et relus dans une dizaine d'années ou seront-ils voués à l'oubli ?

\section{Bibliographie}

1)

\section{Corpus :}

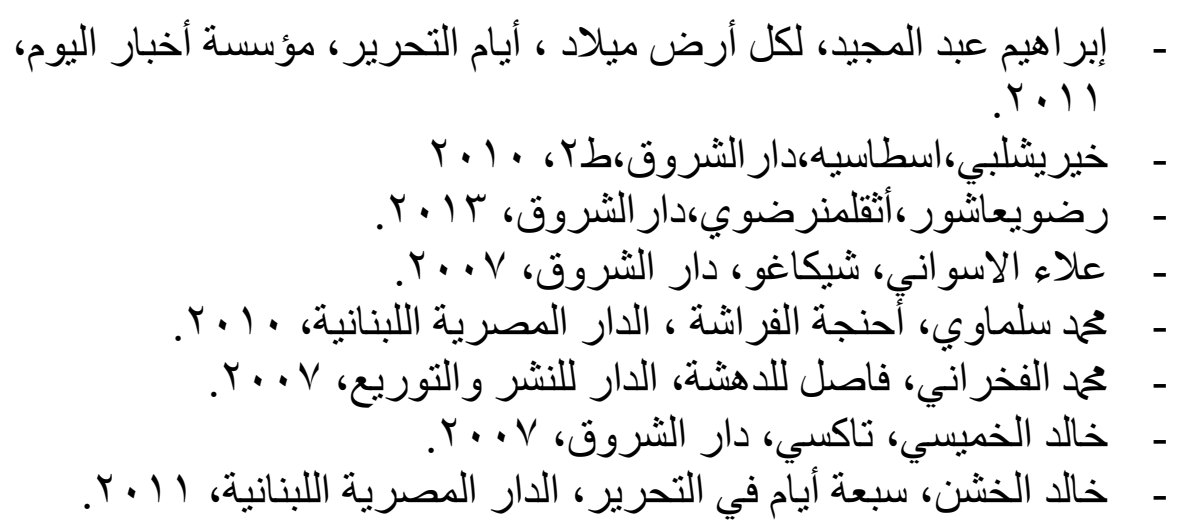

2) Des ouvres écrites sur le « printemps arabe»

- Amin, Samir, Le monde arabe dans la longue durée. Le «Printemps » arabe, Paris, Le Temps des Cerises, 2011.

- Ben Jelloun, Tahar, Par le feu : récit, Paris, Gallimard, 2011.

- Bauchard, Denis, Le nouveau monde arabe. Enjeux et instabilités, Bruxelles, André Versaille, 2012.

- Camus, Albert, L'homme révolté, Gallimard, Collection "Folio essais", 1951.

- Charaudeau, Patrick (2011), Les médias et l'information. L'impossible transparence $d u$ discours, Paris : Ina Editions. 
- Discours télévisés du président Hosni Moubarak sur la chaîne satellitaire étatique Al-Masrya, 28 janvier 2011, 01 et 10 février 2011

- Dominique Fisher,Écrire l'Urgence Assia Djebar et Tahar Djaout, Paris, L'Harmattan, 2008.

- El Aswani, Alaa, Chroniques de la révolution égytienne; traduit de l'arabe, Égypte par Gilles Gauthier, Arles : Actes Sud, 2011.

- Guibal, Claude et Salaun, Tangi, L'Égypte de Tahrir, Anatomie d'une révolution, Paris, Seuil, 2011.

- Guidère, Mathieu, Le choc des révolutions arabes, Paris, Autrement, 2011.

- Ibn-Manzur Lisān al- al-'Arabe al-muhịịt, Beyrouth, Dār Șādir, 1980, 1913.

- Ireton, François, Battesti, Vincent (dir.), L'Égypte au présent, inventaire d'une société avant révolution, Paris, Sindbad Actes Sud, collection Bibliothèque arabe : Hommes et société, 2011.

- Mazeau, Guillaume, Moisand, Jeanne, « Révolution et crise de la temporalité ». Entretien avec Yves Citton et Myriam Revault d'Allonnes», La Vie des idées, [En ligne], mis en ligne le 12 mars 2013, consulté le 29 aout 2013, URL : http://www.laviedesidees.fr/Revolution-etcrise-de-la.html.

- Nassef, Mohamed Abdel-Hafez. (2012) «L’Égypte du 25 Janvier vue par la presse internationale ». Le progrès égyptien, p.4.

- Osman, Tarek, Révolutions égyptiennes de Nasser à la chute de Moubarak, Paris, Les Belles Lettres, collection Le bruit du monde, 2011.

- Ricoeur, Paul, «La crise : un phénomène spécifiquement moderne ? », Revue de théologie et de philosophie, [En ligne], $2008 \mathrm{n}^{\circ} 120$, consulté le 01 septembre 2013, URL : http://www.fondsricoeur.fr/photo/crise\%284\%29.pdf 
- Souif, Ahdaf, Cairo: my city, our revolution, Londres, Bloomsbury Publishing, 1ère éd. 2012.

- S. Boussois, Le Moyen Orient à l'aube du Printemps arabe, Bruxelles, Editions du Cygne, 2011.

- Vincent Battesti, François Ireton (dir.) L'Égypte au présent. Inventaire d'une société avant révolution, Arles, Actes Sud, 2011 (http://anthropoasis.free.fr/spip.php?article577)

- Xavier Luffin, Printemps arabe et littérature, de la réalité à la fiction, de la fiction à la réalité, L'Aurore, 2012.

3) Ouvrages sur le genre littéraire et

\section{l'écriture d'urgence :}

- Combe, Dominique (1992), Les Genres littéraires, Paris, Hachette

- Dominique Fisher, Écrire l'urgence, Assia Djebar et Tahar Djaout, Paris, L'Harmattan, coll. «Études transnationales, francophones et comparées », 2008.

- Genette, Gérard (1991). Fiction et diction. Paris: Seuil.

- Hamburger, Käte (1977). Logique des genres littéraires. Paris: Seuil, 1986.

- Jean-François Soulet, L'Histoire immédiate, Paris, PUF, coll. «Que sais-je ? », n²841, 1994.

- Jean-Philippe Toussaint, L'urgence et la patience, Paris, Les Éditions de Minuit, 2012.

- Kerbrat-Orecchioni, Catherine (2001), Les Actes de langage dans le discours, Paris, Nathan.

- Lejeune, Philippe (1975), Le Pacte autobiographique, Paris, Seuil.

- Macdonald, Margaret, (1989), «Le Langage de la fiction », Poétique, 78, 219-235.

- Maugiron, Nathalie et Ibrahim, Gamal (2000) «Pouvoir de la censure ou censure du pouvoir ». EMA revues, Égypte, monde arabe, no.3. pp. 125- 148. 
- OSMAN, Tarek, Révolutions égyptiennes de Nasser à la chute de Moubarak, Paris, Les Belles Lettres, coll. Le bruit du monde, 2011.

- Platon. La République, trad. Pierre Pachet. Paris: Folio/Essais, 1993.

- Ricœur, Paul, La Mémoire, l'histoire, l'oubli, Paris, Seuil, coll. «L'ordre philosophique », 2000.

- Schaeffer, Jean-Marie (1989). Qu'est-ce qu'un genre littéraire?, Collection Poétique, Paris: Seuil.

- Saul, Samir (2012) «La crise économique internationale de 2008 a-t-elle favorisé l'avènement du " printemps » arabe ? Le cas de l'Égypte », Communication ACSLAF.

- Schaeffer, Jean-Marie (1986). Théorie des genres, ouvrage collectif. Paris: Seuil, Points.

- Schabert, Ina (1982), «Fictional Biography, Factual Biography, and their Contaminations », Biography, 5, 1, 1-16.

- Van Dijk, T.A (2004) « Politique, idéologie et discours », SEMEN, No.21, pp. 278-316.

4)

\section{Resources électroniques:}

http://www.lexpressiondz.com/chroniques/a nalyses_du_professeur_chitour/138532

- $\quad$ http://digital.ahram.org.eg/articles.aspx?Seri $\underline{\mathrm{al}=571903 \& \mathrm{eid}=460}$

- $\quad$ http://www.arretsurimages.net/vite.php?id=9 $\underline{125}$

http://hebdo.ahram.org.eg/Archive/2012/7/1 1/doss2.htm Mohamed Abdel Qodous, Al Ahram hebdo. http://www.laviedesidees.fr/Revolution-etcrise-de-la.html

- $\quad$ http://www.laviedesidees.fr/Revolution-etcrise-de-la.html

- http://www.fondsricoeur.fr/photo/crise\%284\%29.pdf 